\title{
Female preclinical presenilin-1 mutation carriers unaware of their genetic status have higher levels of depression than their non-mutation carrying kin
}

\author{
J M Ringman, C Diaz-Olavarrieta, Y Rodriguez, M Chavez, F Paz, J Murrell, M Angel Macias, \\ M Hill, C Kawas
}

J Neurol Neurosurg Psychiatry 2004;75:500-502. doi: 10.1136/jnnp.2002.005025

\begin{abstract}
Objectives: To study depressive symptoms in preclinical presenilin-1 (PS1) related Alzheimer's disease.

Methods: Participants were 33 Mexican women at risk for inheriting PS1 mutations who were not demented. They were interviewed, underwent cognitive testing, and completed the Beck depression inventory (BDI). PSI mutation status was determined. Mean BDI scores were compared between PS1 mutation carriers and non-carriers. The percentage of subjects who reported seeing a psychiatric professional, and the percentage complaining of memory loss were compared between groups. Regression analysis was used to determine whether mutation status predicted BDI scores after adjusting for age, education, mini-mental state examination, and subjective memory function.

Results: PS1 mutation carriers $(n=17)$ scored significantly higher than non-carriers $(n=16)$ on the BDI (mean score, $14.4 v 6.5, p=0.017$ ); $24 \%$ of mutation carriers and $12.5 \%$ of non-carriers admitted having sought help from a psychiatric professional (NS). Mutation status remained a significant predictor of BDI scores after adjusting for potential covariates. Though not demented, mutation carriers tended to score lower than non-carriers on several neuropsychological tests.

Conclusions: Depressive symptoms can occur early in the course of PS1 related Alzheimer's disease, at least in women. This supports the hypothesis that depression may occur as a direct result of the neuropathology underlying Alzheimer's disease.
\end{abstract}

$\mathrm{D}$ epression is common in Alzheimer's disease ${ }^{1}$ and can be an early or even prodromal symptom. ${ }^{23}$ As the neuropathology of Alzheimer's disease can precede symptoms by decades, ${ }^{45}$ early mood changes may represent a direct manifestation of this pathology. The relation between depression and incipient dementia, however, is complex and difficult to study. Knowing who will and will not develop Alzheimer's disease would help us establish early behavioural changes occurring in this disorder. Families in whom Alzheimer's disease is inherited owing to identifiable mutations in the presenilin-l (PS1) gene provide such an opportunity.

Retrospective case-control ${ }^{26-9}$ and prospective studies ${ }^{2}{ }^{6}$ 8-11 indicate an association between depression and the subsequent increased risk of dementia. Though these findings may be interpreted as suggesting that depression is a sign of incipient dementia, they could also indicate that it occurs as a reaction to cognitive decline, is a risk factor or a surrogate marker for a risk factor for Alzheimer's disease, or decreases the threshold for the manifestation of Alzheimer symptoms. By studying persons with pathogenic mutations in the PSI gene in the preclinical phase we may control for many of these variables.

Observations in families in which Alzheimer's disease is inherited as a genetic trait ${ }^{12-16}$ have suggested that depression occurs often and may appear before cognitive symptoms. However, mood changes in preclinical carriers of determinant mutations have not been studied systematically.

We undertook a cross sectional study examining the effect of PS1 mutations on mood by comparing scores on a self rated questionnaire of depressive symptoms between related mutation carriers and non-carriers unaware of their genetic status, who were in the preclinical phase of the illness.

\section{METHODS}

\section{Subjects}

Subjects were from 10 Mexican families featuring one of two distinct PS 1 mutations. Nine of the 10 families have the same A431E substitution ${ }^{17}$ in the PSI protein. This mutation was associated with the neuropathology of Alzheimer's disease in another Mexican-American kindred. ${ }^{18}$ As mutation carriers within these families all have the same dinucleotide repeat alleles flanking the PSI gene, this probably represents a founder effect. ${ }^{19}$ The 10th family has an unreported mutation causing an L235V substitution in PS1.

Neuropsychological testing had been undertaken on some subjects by $\mathrm{CDO}$ and $\mathrm{YR}^{20}$ who contacted them and their relatives for participation in the present study. In all, 70 subjects were examined. Subjects were excluded if they had functional loss as the result of cognitive decline $(n=8)$ or if their mini-mental state examination (MMSE) score was below $25(\mathrm{n}=3)$. Subjects were also excluded if they were at or beyond the mean age of dementia diagnosis in their family. This was 43 years for the A431E mutation (excluding nine subjects) and 48 for the L235V mutation (excluding no subjects). Fifty subjects (33 female, 17 male) remained. Because of the relative paucity of male volunteers (none in family L235V) and the potential incomparability of Beck depression inventory (BDI) scores between male and female subjects, ${ }^{21}$ we limited our analyses to women (33 subjects).

Subjects were examined at the Instituto Nacional de Neurologia y Neurocirugia (INNN) in Mexico City or in their homes. Written informed consent was obtained from all the participants. During the consent process subjects were told that their blood would be tested for a mutation causing early onset Alzheimer's disease, which they were at a 50\% chance

Abbreviations: $B D I$, Beck depression inventory; MMSE, mini-mental state examination; WAIS, Wechsler adult intelligence scale 
of carrying, and that they would not be told the result. This protocol was approved by the institutional review boards at the University of California, Irvine, the INNN, and the Universidad de Guadalajara.

\section{Cognitive testing}

Subjects underwent an interview and a battery of Spanish cognitive tests ${ }^{20}$ which included the BDI. ${ }^{22}$ The interview also included questions regarding previous treatment by psychiatric professionals and about whether subjects felt they had problems with their memory or thinking.

Subjects completed the written form of the BDI. This is a standardised, validated, ${ }^{22}$ self administered survey consisting of 21 questions, on each of which subjects rate their depressive symptoms. By totalling the score on each item, the BDI provides a measure of depression severity.

Blood was drawn for genetic testing at the time of these assessments. Investigators were thus blinded to the genetic status of subjects at the time of both administration and scoring of the BDI.

\section{Genetic testing}

The blood was sent to JM who isolated the genomic DNA. The presence or absence of the A431E mutation was determined by restriction fragment length polymorphism analysis, and the L235V mutation by sequencing of exon 8 of the PSI gene.

\section{Data analysis}

All results were collected by the principal investigator (JR). Mutation carriers and non-carriers were compared with regard to mean age, years of education, BDI scores, and neuropsychological test scores by independent samples, separate variance $t$ tests. The square root of BDI scores was used in analyses. Two way analysis of variance (ANOVA) was employed to study differences in BDI scores attributed to mutation status and PSI mutation type.

A $\chi^{2}$ test was also used to compare the proportion of mutation carriers and non-carriers who answered yes when asked if they felt they had memory problems. Regression models were used to explore the interrelations of mutation status, subjective memory loss, age, education, and MMSE score.

\section{RESULTS}

The demographic characteristics of the study population are given in table 1 . Mean age $(30.8 v 29.1$ years, $\mathrm{p}=0.56)$ and years of education (11.6 $v 12.5, \mathrm{p}=0.42)$ did not differ between PS1 mutation carriers $(n=17)$ and non-carriers $(n=16)$. When a probability $(p)$ value of $<0.05$ was used as a cut off, PSI mutation carriers performed more poorly on the MMSE, trails making test part B (time), WMS associative learning subtest-immediate recall, and the Wechsler adult intelligence scale (WAIS) block design.

Mutation carriers scored significantly higher on the BDI than non-carriers ( $14.4 v 6.5, \mathrm{p}=0.017)$ (fig 1). Thirty five per cent of mutation carriers and $25 \%$ of non-carriers had BDI scores above 12. Twenty four per cent of mutation carriers admitted to having sought help from a psychologist or psychiatrist, compared with $12.5 \%$ of non-carriers.

There were 22 subjects from families with the A431E mutation ( 12 carriers and 10 non-carriers) and 11 from the family with the L235V mutation (five carriers and six noncarriers). In families featuring the A431E mutation, the mean BDI score of the mutation carriers was 8.6, $v 2.5$ for noncarriers; for the family with the L235V mutation the mean score of the mutation carriers was 16.8, $v 8.9$ for non-carriers. Two way ANOVA indicated significant differences in mean BDI scores between mutation carriers and non-carriers
Table 1 Demographic and cognitive characteristics of the study population

\begin{tabular}{|c|c|c|c|}
\hline & $\begin{array}{l}\text { PS1 mutation } \\
\text { present } \\
(\mathrm{n}=17)\end{array}$ & $\begin{array}{l}\text { PS1 mutation } \\
\text { absent } \\
(n=16)\end{array}$ & p Value \\
\hline Age (years) & $30.8(1.87)$ & $29.1(2.04)$ & 0.558 \\
\hline Education (years) & $11.6(0.65)$ & $12.5(0.81)$ & 0.418 \\
\hline memory complaints & $9(53 \%)$ & 9 (56\%) & 0.583 \\
\hline BDI score & $14.4(3.10)$ & $6.5(1.66)$ & $0.017^{*}$ \\
\hline Number with $B D I$ score $\geqslant 13$ & $6(35 \%)$ & $4(25 \%)$ & 0.397 \\
\hline $\begin{array}{l}\text { Number having sought help } \\
\text { from a psychiatric professional }\end{array}$ & $4(24 \%)$ & $2(12.5 \%)$ & 0.616 \\
\hline $\begin{array}{l}\text { MMSE score } \\
\text { Trails making test-part B }\end{array}$ & $28.0(0.31)$ & $29.4(0.18)$ & 0.001 \\
\hline $\begin{array}{l}\text { time (s) } \\
\text { Wechsler memory scale, }\end{array}$ & $125.3(13.33)$ & 72.8 & 0.001 \\
\hline $\begin{array}{l}\text { associative learning } \\
\text { immediate recall }\end{array}$ & 16.0 & 18. & 0.027 \\
\hline WAIS block design & $31.7(1.79)$ & $36.7(1.55)$ & 0.044 \\
\hline
\end{tabular}

Values are mean (SD) or $\mathrm{n}(\%)$

$\mathrm{BDI}$, Beck depression inventory; MMSE, mini-mental state examination;

PS1, presenilin-1; WAIS, Wechsler adult intelligence scale.

$(p=0.022)$. Differences between the two types of mutation (L235V v A43lE) almost reached significance $(p=0.054)$.

Fifty three per cent of mutation carriers had subjective memory problems, compared with $56 \%$ of non-carriers $(\mathrm{p}=0.583)$. Adding mutation status (presence or absence) to a regression model with subjective memory problems as a predictor increased multiple $R^{2}$ from 0.07 to 0.25 . When mutation status was added to a model including age, education, and MMSE as predictors, multiple $R^{2}$ increased from 0.04 to 0.19 .

\section{DISCUSSION}

The results of our study are consistent with reports that depression occurs in at-risk members of families with early onset Alzheimer's disease. ${ }^{12-16}$ We divided these subjects into those carrying and not carrying PSI mutations and found that women with mutations had higher levels of depression as measured on the BDI. As subjects were unaware of their mutation status, and as this continued to be a strong predictor of BDI scores when the presence or absence of cognitive complaints was taken into account, it is unlikely

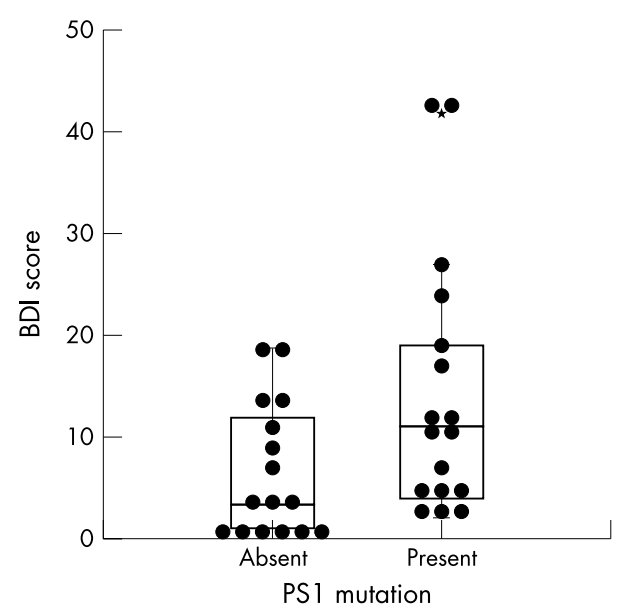

Figure 1 Beck depression inventory (BDI) total scores for 33 Mexican female preclinical PS1 mutation carriers $(n=17)$ and non-carriers $(n=16)$. 
that this represents reactive depression to perceived memory loss. It should be noted that both the PS I mutation positive subjects with the highest scores on the BDI (42) scored 30/30 on the MMSE, denied any cognitive decline, and were 8 and 23 years younger than the mean age of Alzheimer's disease diagnosis in their families.

The BDI is often used to measure depression and has been translated into various languages and validated in many cultures. ${ }^{22}$ A drawback of its use in Alzheimer's disease is that, as a self rated scale, it may underestimate depression because of the loss of insight that occurs in this illness. ${ }^{23}$ However, impaired awareness of one's deficits increases as Alzheimer's disease advances ${ }^{24}$ and we therefore feel that the BDI is a valid instrument for measuring depression in these presymptomatic or mildly affected subjects.

This population allows us to study the relation between levels of depression and the onset of cognitive decline. The PSI mutation carriers in our study performed more poorly than average on several neuropsychological tests. Though some mutation carriers were therefore likely to be in the initial stages of cognitive decline, none had yet experienced deficits in social or occupational functioning and did not meet criteria for dementia.

Our population, in which subjects are at a 50\% risk of carrying a mutation determinant for Alzheimer's disease, has the advantage of allowing the relation between depression and early Alzheimer's disease to be studied in a relatively small number of subjects who are well matched for socioeconomic variables. In addition, studying younger subjects controls for confounding factors such as concurrent medical illness and uncertainty over the pathological substrate of the dementing illness. On the other hand, the generalisability of the results of our study is limited by the unique nature of the population. In particular, there are some clinical differences between PSI related Alzheimer's disease and sporadic late onset Alzheimer's disease. The study does, however, provide additional evidence that depression can be an early symptom heralding the onset of Alzheimer's disease.

\section{ACKNOWLEDGEMENTS}

This study was supported by Alzheimer's Association Grant NIRG 01-2797.

\section{Authors' affiliations \\ J M Ringman, Alzheimer's Disease Center, UCLA Department of Neurology, Los Angeles, California, USA \\ M Hill, C Kawas, Institute for Brain Aging and Dementia, Department of Neurology, University of California, Irvine, CA \\ C Diaz-Olavarrieta, Y Rodriguez, M Chavez, F Paz, Instituto Nacional de Neurologia y Neurocirugia, Mexico City, Mexico \\ J Murrell, Department of Pathology and Laboratory Medicine, Indiana University, Indianapolis, IA \\ M Angel Macias, Neurosciences Department, CUCS, Universidad de Guadalajara, Mexico}

Competing interests: none declared
Correspondence to: Dr John M Ringman, Alzheimer's Disease Center, UCLA Department of Neurology, 710 Westwood Plaza, Suite 2-238, Los Angeles, CA 90095-1769, USA; jringman@mednet.ucla.edu

Received 8 October 2002

In revised form 10 July 2003

Accepted 12 July 2003

\section{REFERENCES}

1 Mega MS, Cummings JL, Fiorello T, et al. The spectrum of behavioral changes in Alzheimer's disease. Neurology 1996:46:130-5.

2 Wetherell JL, Gatz M, Johansson B, et al. History of depression and other psychiatric illness as risk factors for Alzheimer disease in a twin sample. Alzheimer Dis Assoc Disord 1999;13:47-52.

3 Berger AK, Fratiglioni L, Forsell Y, et al. The occurrence of depressive symptoms in the preclinical phase of $A D$ : a population-based study. Neurology 1999;53:1998-2002.

4 Braak H, Braak E. Neuropathological staging of Alzheimer-related changes. Acta Neuropathol 1991;82:239-59.

5 Troncoso JC, Cataldo AM, Nixon RA, et al. Neuropathology of preclinical and clinical late-onset Alzheimer's disease. Ann Neurol 1998;43:673-6.

6 Jorm AF, van Duiin CM, Chandra V, et al. Psychiatric history and related exposures as risk factors for Alzheimer's disease: a collaborative re-analysis of case-control studies. EURODEM Risk Factors Research Group. Int J Epidemiol 1991;20:S43-7.

7 Agbayewa MO. Earlier psychiatric morbidity in patients with Alzheimer's disease. J Am Geriatr Soc 1986;34:561-4.

8 Shalat SL, Seltzer B, Pidcock C, et al. Risk factors for Alzheimer's disease: a case-control study. Neurology 1987; 37:1630-3.

9 Kokmen E, Beard CM, Chandra V, et al. Clinical risk factors for Alzheimer's disease: a population-based case-control study. Neurology 1991;41:1393-7.

10 Reding M, Haycox J, Blass J. Depression in patients referred to a dementia clinic. A three-year prospective study. Arch Neurol 1985;42:894-6.

11 Devanand DP, Sano M, Tang MX, et al. Depressed mood and the incidence of Alzheimer's disease in the elderly living in the community. Arch Gen Psychiatry 1996;53:175-82.

12 Kennedy AM, Newman S, McCaddon A et al. Familial Alzheimer's disease. A pedigree with a missense mutation in the amyloid precursor protein gene (amyloid precursor protein 717 valine $\rightarrow$ glycine). Brain 1993;1 16:309-24.

13 Lopera F, Ardilla A, Martinez A, et al. Clinical features of early-onset Alzheimer disease in a large kindred with an E280A presenilin-1 mutation. JAMA 1997:277:793-9.

14 Lowenberg K, Waggoner RW. Familial organic psychosis (Alzheimer's type). Arch Neurol Psychiatry 1934;31:737-54

15 Heston LL, Lowther DLW, Levanthal CW. Alzheimer's disease: a family study. Arch Neurol 1966:15:225-33

16 Feldman RG, Chandler KA, Levy LL, et al. Familial Alzheimer's disease. Neurology 1988;13:14-19.

17 Ringman JM, Jain V, Murrell J, et al. A novel Ala431Glu mutation in Presenilin-1 associated with familial early-onset Alzheimer's disease [abstract]. Hum Genet 2001;109:242.

18 Cochran EJ, Murrell JR, Fox J, et al. A novel mutation in the Presenilin-1 gene (A431E) associated with early-onset Alzheimer's disease [abstract]. J Neuropathol Exp Neurol 2001;60:544.

19 Murrell JR, Faber K, Alonso ME, et al. The A431E Presenilin 1 gene mutation associated with familial Alzheimer's disease in individuals of Mexican descent: evidence for a founder effect [abstract]. I Neuropathol Exp Neurol 2003;62:543.

20 Diaz-Olavarrieta C, Ostrosky-Solis F, Garcia de la Cadena C, et al. Neuropsychological changes in subjects at risk of inheriting Alzheimer's disease. Neuroreport 1997;8:2449-53

21 Salokangas RKR, Vaahtera K, Pacriev S, et al. Gender differences in depressive symptoms. An artefact caused by measurement instruments? $J$ Affect Disord 2002;68:215-20.

22 Jurado S, Villegas E. La estandarizacion del Inventario de Depresion de Beck para los residentes de la ciudad de Mexico. Salud Mental 1998;21:26-31.

23 Wagle AC, Ho LW, Wagle SA, et al. Psychometric behaviour of BDI in Alzheimer's disease patients with depression. Int J Geriatr Psychiatry 2000;15:63-9.

24 Starkstein SE, Chemerinski E, Sabe L, et al. Prospective longitudinal study of depression and anosognosia in Alzheimer's disease. Br J Psychiatry 1997;171:47-52. 